\title{
Pipeline pressure distribution finding methods
}

\author{
Pyanylo Ya., Sobko V. \\ Centre for Mathematical Modelling of Pidstryhach Institute \\ for Applied Problems of Mechanics and Mathematics, \\ National Academy of Sciences of Ukraine \\ 15 Dudayev str., 79000, Lviv, Ukraine
}

(Received 1 December 2016)

\begin{abstract}
The method of solving problems of mathematical physics, in particular for calculating a non-stationary gas flow in pipelines, is proposed in this article on the basis of the biorthogonal polynomial constructed by the authors. The method of solving the problem by means of the separation of variables in the base of biorthogonal polynomials is investigated. The analytical-approximate and approximate solutions of the problem as the sum of some biorthogonal and quasi-spectral polynomials are found. The comparative analysis between the obtained analytical-approximate and approximate solutions is conducted. The influence of parameters of methods, including the order of the partial sum, a bit grid, and an accuracy error of calculations on the obtained solution are studied. The results of calculation are presented in the form of tables.
\end{abstract}

Keywords: spectral methods, mathematical model, non-stationary gas flow, linearization, biorthogonal and quasi-orthogonal polynomials.

2000 MSC: $12 \mathrm{E} 10$

UDC: 519.6:539.3

\section{Introduction}

Spectral methods are used both in theoretical studies and for solving a wide class of problems of mathematics and mechanics. Their essence is that the functions included into the model are presented in the form of orthogonal series in accordance to the selected basis. Finding the solution is reduced to calculating the coefficients of orthogonal series of the desired solution. It is shown $[6,7]$ that the choice of orthogonal basis one should coordinate with the domain of definition of the desired solution. The positive sides are those that many orthogonal bases are studied well enough, they are easy to use, and the solving algorithms constructed on their basis are easy for automation. The negative side is that the summation of corresponding series is, as a rule, an incorrect problem. Therefore, not all the criteria to the solutions of problems one can satisfy by means of using of one orthogonal basis. Due to the fact, we are modifying the existing bases or constructing a new one to meet the broader criteria. One of the methods of consideration of these comments is the usage of biorthogonal bases. Nowadays, there are a few papers devoted to their research and practical application. This is mainly due to the fact that formation of these biorthogonal bases is connected with significant difficulties of calculation and they are not studied enough.

\section{Formulation of the problem}

The system of the interconnected differential equations in partial derivatives is the common mathematical model of gas flow in the pipeline in the isothermal case

$$
\left\{\begin{array}{l}
\frac{\partial p(y, t)}{\partial y}+\alpha \rho \frac{\partial}{\partial y}\left(\frac{v^{2}(y, t)}{2}\right)+\rho g \frac{\partial h(y, t)}{\partial y}+\frac{\lambda \rho v^{2}(y, t)}{2 D}+\frac{\partial(\rho v(y, t))}{\partial t}=0 \\
\frac{\partial(\rho v(y, t))}{\partial y}+\frac{1}{c^{2}} \frac{\partial p(y, t)}{\partial t}=0
\end{array}\right.
$$


where $\omega=\rho v$ is the mass gas consumption (gas velocity); $A$ is the sound velocity in gas; $\alpha$ is the Coriolis coefficient; $\rho, v, p$ are the density, the gas velocity and the gas pressure accordingly; $\lambda$ is the hydraulic resistance coefficient; $D$ is the diameter of the pipeline; $g$ is gravity acceleration; $h$ is the relative height of the pipeline location; $t>0$ is time; $y \in[0, l]$ is the line coordinate, $l$ is the length of the pipeline.

In practice, the limiting conditions are formulated on the input and output of compressor stations. As usual, the stationary pressure distribution is the initial state at the beginning of the non-stationary process. Therefore, the problem of mathematical physics in this case is as follows.

One can find the solution of the system (1) in accordance to the initial stationary pressure distribution

$$
p(y, 0)=\sqrt{p_{0}^{2}-\frac{\lambda z R T}{D}\left(\frac{\rho_{s} q_{s}}{s}\right)^{2} y}
$$

and limiting conditions on volumetric gas consumption

$$
\begin{gathered}
q_{0}(t)=q_{0 n}+\left(q_{0}-q_{0 n}\right) e^{-\gamma_{0} t} ; \\
q_{l}(t)=q_{l n}+\left(q_{l}-q_{l n}\right) e^{-\gamma_{l} t},
\end{gathered}
$$

stand on inputs and outputs of the pipeline correspondingly.

In this case $p_{0}$ is the value of pressure on the pipeline start; $\rho_{s}, q_{s}$ are the values of density and volumetric consumption in standard conditions, $s=\pi D^{2} / 4 ; q_{0}, q_{0 n}$ are the volumetric gas consumptions at the initial and new states of gas flow and the parameter $\gamma_{0}$, which characterizes velocity of transition from one state into other on the pipeline start; $q_{l}, q_{l n}, \gamma_{l}$ are the the similar parameters at the pipeline end. In the case of transition to mass velocity the limiting conditions are the following [9]:

$$
\omega_{0}=\omega(0, t)=\frac{\rho_{s}}{s} q_{0}(t), \quad \omega_{l}=\omega(l, t)=\frac{\rho_{s}}{s} q_{l}(t) .
$$

\section{Linearization of the output nonlinear system}

The linearised variant of the system (1) [9] has the form

$$
\left\{\begin{array}{l}
A_{0} \frac{\partial p(y, t)}{\partial y}+A_{1} \frac{\partial \omega(y, t)}{\partial y}+\frac{\partial \omega(y, t)}{\partial t}+A_{2} \omega(y, t)+A_{3} p(y, t)=-A_{4} \\
\frac{\partial \omega(y, t)}{\partial y}+\frac{1}{c^{2}} \frac{\partial p(y, t)}{\partial t}=0
\end{array}\right.
$$

where

$$
\begin{gathered}
a_{p}=p_{1}\left(1+f p_{1}\right)-b_{p} p_{1}, \quad c_{0}=1-\alpha v_{c}^{2} \rho_{0} T_{0} b_{p} /\left(p_{0} T\right), \quad c_{1}=\alpha v_{c}, \quad a_{v}=v_{1}+v_{2}, \\
b_{v}=-v_{1} v_{2}-\frac{1}{8}\left(v_{2}-v_{1}\right)^{2}, \quad c_{2}=\alpha b_{v} /(2 D), \quad c_{3}=\frac{\rho_{0} T_{0}}{p_{0} T} b_{p}\left(g \frac{\delta h}{\delta y}+\frac{\lambda a_{v}}{2 D}\right), \\
c_{4}=\frac{\rho_{0} T_{0}}{p_{0} T} a_{p}\left(g \frac{\delta h}{\delta y}+\frac{\lambda a_{v}}{2 D}\right), \quad p \in\left[p_{1}, p_{2}\right],
\end{gathered}
$$

$p_{1}$ and $p_{2}$ stand for the limits of pressure change, $v_{A}$ is the average volume of gas flow in the pipeline, which is accepted as known, $v_{1}$ and $v_{2}$ are the limits of change of gas flow velocity. For calculation of the coefficient of pressure $z$, which describes difference of the real gas from the ideal gas, one can use the empirical formula $z=1 /(1+f p)$, where $p$ is measured in atmospheres, and $f=\left(24-0.21 t^{\circ} \mathrm{C}\right) \cdot 10^{-4}$, $t^{\circ} \mathrm{C}$ is the temperature of gas Celcius; $R$ is the gas constant.

We can note that initial distribution of pressure (2) is obtained in the stationary case from the nonlinear system of differential equations. Therefore, it is necessary to have its parametrical image in 
the stationary case for finding the correct solution of the system (6). Thus, the problem of mathematical physics is as follows: to find the solution of the system (6) according to the initial (2) and the boundary (5) conditions.

\section{Analytical-approximate solution}

We will consider the horizontal pipelines without taking into account the Coriolis force at the constant value of the pressure coefficient, that is $\alpha=0$ and $c_{0}=1, c_{1}=0, c_{2}=0$ correspondingly. Then we write the system (6) in the form

$$
\left\{\begin{array}{l}
\frac{\partial p(y, t)}{\partial y}+\frac{\partial \omega(y, t)}{\partial t}+A_{3} p(y, t)=-A_{4} \\
\frac{\partial \omega(y, t)}{\partial y}+\frac{1}{c^{2}} \frac{\partial p(y, t)}{\partial t}=0
\end{array}\right.
$$

Since the length of the pipeline is $l$, that is $0 \leqslant y \leqslant l$, and functions which help us to solve this problem are considered on an interval $[-1,1]$, then we change

$$
y=\frac{l(x+1)}{2}, \quad w(y, t)=w\left(\frac{l(x+1)}{2}, t\right)=W(x, t), \quad p(y, t)=p\left(\frac{l(x+1)}{2}, t\right)=P(x, t) .
$$

Using the formulas (8), we write the system (7) and the conditions (2), (5) in the form

$$
\begin{aligned}
& \left\{\frac{2}{l} A_{0} \frac{\partial P(x, t)}{\partial x}+\frac{\partial W(x, t)}{\partial t}+A_{3} P(x, t)=-A_{4},\right. \\
& \left\{\frac{2}{l} \frac{\partial W(x, t)}{\partial x}+\frac{1}{c^{2}} \frac{\partial P(x, t)}{\partial t}=0,\right. \\
& P(x, 0)=\sqrt{p_{0}^{2}-\frac{\lambda z R T}{D}\left(\frac{\rho_{s} q_{s}}{s}\right)^{2} \frac{l(x+1)}{2}}, \\
& W_{0}=W(-1, t)=\frac{\rho_{s}}{s} q_{0}(t), \quad W_{l}=W(1, t)=\frac{\rho_{s}}{s} q_{l}(t) .
\end{aligned}
$$

We can obtain the following from the system (9) and conditions (10), (11)

$$
\frac{4 c^{2}}{l^{2}} \frac{\partial W^{2}(x, t)}{\partial x^{2}}-\frac{\partial^{2} W(x, t)}{\partial t^{2}}+\frac{2 c^{2} A_{3}}{l} \frac{\partial W(x, t)}{\partial x}=0 .
$$

Even if

$$
W(x, t)=V(x) e^{\beta x} G(t),
$$

where $\beta$ is the unknown parameter, which will be determined in the result of solving; $V(x)$ are basis functions, which have the right ratios

$$
\begin{aligned}
& \frac{d^{2} V_{i}^{n+\bar{i}}(x)}{d x^{2}}=-\frac{V_{i}^{n+\bar{i}}(x)}{\lambda_{i+(-1)^{\bar{i}+1}}^{n}}+\frac{\tau_{i+(-1)^{\bar{i}+1}}^{n}}{\lambda_{i+(-1)^{\bar{i}+1}}^{\prime}} T_{n+1+\bar{i}}^{\prime}(x), \quad i=1, \ldots, n, \\
& \frac{d^{2} V_{n+1}^{n+1}(x)}{d x^{2}}=\frac{(n+2)^{2} \pi}{8} \sum_{k=1}^{\frac{n}{2}} \frac{\bar{c}_{2}^{2 k}}{\lambda_{2 k}^{n}} \frac{V_{2 k-1}^{n+1}(x)}{N_{2 k-1}^{n}}+\frac{1}{15} n(n+1)(n+3)(n+4) T_{n+2}^{\prime}(x), \\
& \frac{d^{2} V_{n+2}^{n}(x)}{d x^{2}}=\frac{(n+1)^{2} \pi}{2} \sum_{k=1}^{\frac{n}{2}} \frac{\bar{c}_{1}^{2 k-1}}{\lambda_{2 k-1}^{n}} \frac{V_{2 k}^{n}(x)}{N_{2 k}^{n}}+\frac{1}{15}(n-1) n(n+2)(n+3) T_{n+1}^{\prime}(x),
\end{aligned}
$$


where $V_{n+1}^{n+1}(x)=T_{n+2}^{\prime}(x), V_{n+2}^{n}(x)=T_{n+1}^{\prime}(x), \bar{i}=0$ for even values $i$, and $\bar{i}=1$ for uneven values $i . \quad T_{n+1}=T_{n+1}(x)$ and $T_{n+2}=T_{n+2}(x)$ are the Chebyshev polynomials of degree $n+1$ and $n+2, \lambda_{i}^{n}, i=1, \ldots, n$ are the eigenvalues of the integral operator $\pi_{1}^{\infty} L=\pi_{1}^{\infty} \int_{-1}^{x} \int_{-1}^{x_{1}}, U_{2 i}^{2 s}(x)=$ $\sum_{j=1}^{s} c_{2 j}^{2 i} \tilde{T}_{2 j}(x), U_{2 i-1}^{2 s-1}(x)=\sum_{j=1}^{s} c_{2 j-1}^{2 i-1} \tilde{T}_{2 j-1}(x)$ are the eigenvalues of this operator, $\tilde{T}_{j}(x)$ is modified Chebyshev polinomials, $\bar{U}_{2 i}^{2 s}(x)=\sum_{j=1}^{s} \bar{c}_{2 j}^{2 i} T_{2 j}(x), \bar{U}_{2 i-1}^{2 s-1}(x)=\sum_{j=1}^{s} \bar{c}_{2 j-1}^{2 i-1} T_{2 j-1}(x)$ are the eigenvalues of this turned operator,

$$
\begin{gathered}
\tau_{2 i}^{2 s}(x)=\frac{c_{2 s}^{2 i}}{4(2 s+1)(2 s+2)}, \quad \tau_{2 i-1}^{2 s}(x)=\frac{c_{2 s-1}^{2 i-1}}{4(2 s)(2 s+1)}, \\
\bar{\tau}_{2 i}^{2 s}(x)=\frac{\bar{c}_{2 s}^{2 i}}{4(2 s)(2 s+1)}, \quad \bar{\tau}_{2 i-1}^{2 s}(x)=\frac{\bar{c}_{2 s-1}^{2 i-1}}{4(2 s-1)(2 s)},
\end{gathered}
$$

the biorthogonal functions

$$
\begin{gathered}
V_{2 i-\bar{i}}^{n+\bar{i}}(x)=\int_{-1}^{x} U_{2 i-1+\bar{i}}^{n-\bar{i}-(-1)^{\bar{i}}}\left(x_{1}\right) d x_{1}, \quad \bar{V}_{2 i-\bar{i}}^{n+\bar{i}}(x)=-\sqrt{1-x^{2}} \int_{-1}^{x} \frac{\bar{U}_{2 i-1+\bar{i}}^{n-\bar{i}-(-1)^{\bar{i}}}\left(x_{1}\right)}{\sqrt{1-x_{1}^{2}}} d x_{1}, \quad i=1, \ldots, n / 2, \\
N_{i}^{n}=\int_{-1}^{1} \frac{V_{i}^{n+\bar{i}}(x) \bar{V}_{i}^{n+\bar{i}}(x)}{\sqrt{1-x^{2}}} d x, i=1, \ldots, n
\end{gathered}
$$

are the standard of biorthogonal functions $[10,11]$.

Let us substitute (13) into the equation (12) and obtain

$$
\frac{4 c^{2}}{l^{2}} V_{x x}^{\prime \prime}(x) G(t)+\left(\frac{8 c^{2}}{l^{2}} \beta+\frac{2 c^{2} A_{3}}{l}\right) V_{x}^{\prime}(x) G(t)+\left(\frac{4 c^{2}}{l^{2}} \beta^{2} G(t)-G_{t t}^{\prime \prime}(t)+\frac{2 c^{2} A_{3}}{l} \beta G(t)\right) V(x)=0 .
$$

Thence, $\beta=-\frac{l c_{3}}{4}$.

As follows we obtain

$$
\frac{4 c^{2}}{l^{2}} V_{x x}^{\prime \prime}(x) G(t)-\left(\frac{c^{2} A_{3}}{4} G(t)+G_{t t}^{\prime \prime}(t)\right) V(x)=0 .
$$

The product $V(x) G(t)$ one can find in the form

$$
V(x) G(t)=\sum_{i=1}^{n+2} V_{i}^{n+\bar{i}}(x) G_{i}(t) .
$$

Then,

$$
\begin{gathered}
V(x) G^{\prime \prime}(t)=\sum_{i=1}^{n+2} V_{i}^{n+\bar{i}} G_{i}^{\prime \prime}(t) \\
V_{x x}^{\prime \prime}(x) G(t)=\sum_{i=1}^{n} \frac{d^{2} V_{i}^{n+\bar{i}}(x)}{d x^{2}} G_{i}(t)+\frac{d^{2} V_{n+1}^{n+1}(x)}{d x^{2}} G_{n+1}(t)+\frac{d^{2} V_{n+2}^{n}(x)}{d x^{2}} G_{n+2}(t) .
\end{gathered}
$$


From the boundary conditions (11), considering that $V_{i}(-1)=V_{i}(1)=0$, we have

$$
\frac{e^{\frac{l c_{3}}{4}} W(1, t)+e^{-\frac{l c_{3}}{4}} W(-1, t)}{2(n+1)^{2}}=G_{n+2}(t), \quad \frac{e^{\frac{l c_{3}}{4}} W(1, t)-e^{-\frac{l c_{3}}{4}} W(-1, t)}{2(n+2)^{2}}=G_{n+1}(t) .
$$

Expressions (15), (16) we substitute into the equation (14) and obtain the following

$$
\begin{aligned}
\frac{4 c^{2}}{l^{2}}\left(\sum_{i=1}^{n} \frac{d^{2} V_{i}^{n+\bar{i}}(x)}{d x^{2}} G_{i}(t)+\frac{d^{2} V_{n+1}^{n+1}(x)}{d x^{2}} G_{n+1}(\right. & \left.t)+\frac{d^{2} V_{n+2}^{n}(x)}{d x^{2}} G_{n+2}(t)\right) \\
& -\frac{c^{2} A_{3}}{4} \sum_{i=1}^{n+2} V_{i}(x) G_{i}(t)-\sum_{i=1}^{n+2} V_{i}(x) G_{i}^{\prime \prime}(t)=0 .
\end{aligned}
$$

The equation (18) we multiply by $\frac{\bar{V}_{i}(x)}{\sqrt{1-x^{2}}}, i=1, \ldots, n$ and integrate in accordance to $x$ within from -1 to 1 . If using equations (17) one will receive the system from $n$ of the equations of the kind

$$
\begin{aligned}
G_{2 i-\bar{i}}^{\prime \prime}(t) & +\left(\frac{4 c^{2}}{l^{2}} \frac{1}{\lambda_{2 i-1+\bar{i}}^{n}}+\frac{c^{2} A_{3}^{2}}{4}\right) G_{2 i-\bar{i}}(t) \\
& =\frac{c^{2}}{l^{2}} \frac{\pi}{4^{\bar{i}}} \frac{\bar{c}_{\bar{i}+1}^{2 i-1+\bar{i}}}{\lambda_{2 i-1+\bar{i}}^{n}} \frac{1}{N_{2 i-\bar{i}}^{n}}\left[e^{\frac{l c_{3}}{4}} W(1, t)+(-1)^{\bar{i}} e^{-\frac{l c_{3}}{4}} W(-1, t)\right], \quad i=1, \ldots, k, \quad 2 k=n,
\end{aligned}
$$

where $\bar{i}=0$ - for even functions, and $\bar{i}=1-$ for uneven functions.

The system (19) is made from $n$ of non-homogeneous linear equations of the second order with the constant coefficients. The solutions of the characteristic equations will be the numbers $k_{1,2}^{2 i-\bar{i}}=$ $\pm \sqrt{\frac{4 c^{2}}{l^{2}} \frac{1}{\lambda_{2 i-1+\bar{i}}^{n}+\frac{c^{2} A_{3}^{2}}{4}}}$. Since,

$$
\begin{gathered}
q_{0}(t)=q_{0 n}+\left(q_{0}-q_{0 n}\right) e^{-\gamma_{0} t}, \\
q_{l}(t)=q_{l n}+\left(q_{l}-q_{l n}\right) e^{-\gamma_{l} t},
\end{gathered}
$$

then

$$
\begin{aligned}
e^{\frac{l c_{3}}{4}} W(1, t)+(-1)^{\bar{i}} & e^{-\frac{l c_{3}}{4}} W(-1, t) \\
& =\frac{\rho_{s}}{s}\left(q_{l n} e^{\frac{l c_{3}}{4}}+\left(q_{l}-q_{l n}\right) e^{-\gamma_{l} t+\frac{l c_{3}}{4}}+(-1)^{\bar{i}}\left(q_{0 n} e^{-\frac{l c_{3}}{4}}+\left(q_{0}-q_{0 n}\right) e^{-\gamma_{0} t-\frac{l c_{3}}{4}}\right)\right) .
\end{aligned}
$$

Therefore, the following functions will be the solutions of the equations of the system (19)

$$
G_{2 i-\bar{i}}(t)=A_{2 i-\bar{i}} e^{\frac{l c_{3}}{4}}+B_{2 i-\bar{i}} e^{-\gamma_{0} t-\frac{l c_{3}}{4}}+E_{2 i-\bar{i}} e^{-\gamma_{l} t+\frac{l c_{3}}{4}}+D_{2 i-\bar{i}} e^{-\frac{l c_{3}}{4}},
$$

where

$$
\begin{aligned}
& A_{2 i-\bar{i}}=\frac{\frac{\rho_{s}}{s} q_{l n} \frac{c^{2}}{l^{2}} \frac{\pi}{4^{i}} \frac{\bar{c}_{\bar{i}+1}^{2 i-1+\bar{i}}}{\lambda_{2 i-1+\bar{i}}^{n}} \frac{1}{N_{2 i-\bar{i}}^{n}}}{\frac{4 c^{2}}{l^{2}} \frac{1}{\lambda_{2 i-1+\bar{i}}^{n}}+\frac{c^{2} A_{3}^{2}}{4}}, \quad B_{2 i-\bar{i}}=(-1)^{\frac{\bar{i}}{i}} \frac{\frac{\rho_{s}}{s}\left(q_{0}-q_{0 n}\right) \frac{c^{2}}{l^{2}} \frac{\pi}{4^{i}} \frac{\bar{c}_{\bar{i}+1}^{2 i-1+\bar{i}}}{\lambda_{2 i-1+\bar{i}}^{n}} \frac{1}{N_{2 i-\bar{i}}^{n}}}{\gamma_{0}^{2}+\frac{4 c^{2}}{l^{2}} \frac{1}{\lambda_{2 i-1+\bar{i}}^{n}}+\frac{c^{2} A_{3}^{2}}{4}} \\
& E_{2 i-\bar{i}}=\frac{\frac{\rho_{s}}{s}\left(q_{l}-q_{l n}\right) \frac{c^{2}}{l^{2}} \frac{\pi}{4^{i}} \frac{\bar{c}_{i+1}^{2 i+1+\bar{i}}}{\lambda_{2 i-1+\bar{i}}^{n}} \frac{1}{N_{2 i-\bar{i}}^{n}}}{\gamma_{l}^{2}+\frac{4 c^{2}}{l^{2}} \frac{1}{\lambda_{2 i-1+\bar{i}}^{n}}+\frac{c^{2} A_{3}^{2}}{4}}, \quad D_{2 i-\bar{i}}=(-1)^{\frac{\rho_{s}}{s}} \frac{q_{0 n} \frac{c^{2}}{l^{2}} \frac{\pi}{4^{i}} \frac{\bar{c}_{i+1}^{2 i-1+\bar{i}}}{\lambda_{2 i-1+\bar{i}}^{n}} \frac{1}{N_{2 i-\bar{i}}^{n}}}{\frac{4 c^{2}}{l^{2}} \frac{1}{\lambda_{2 i-1+\bar{i}}^{n}}+\frac{c^{2} A_{3}^{2}}{4}} .
\end{aligned}
$$


Thus, we found solutions of the system (9) on the interval $x \in[-1,1]$, namely

$$
\begin{aligned}
W(x, t) & =e^{-\frac{l c_{3}}{4} x} \sum_{i=1}^{n+2} V_{i}^{n+\bar{i}}(x) G_{i}(t), \\
P(x, t) & =-\frac{2 c^{2}}{l} e^{-\frac{l c_{3}}{4} x} \sum_{i=1}^{n+2}\left(-\frac{l c_{3}}{4} V_{i}^{n+\bar{i}}(x)+U_{i-(-1)^{\bar{i}}}^{n-\bar{i}-(-1)^{\bar{i}}}(x)\right) \int_{0}^{t} G_{i}(t) d t+P(x, 0),
\end{aligned}
$$

where $U_{n+2}^{n}(x)=T_{n+2}^{\prime \prime}(x), U_{n+1}^{n-1}(x)=T_{n+1}^{\prime \prime}(x)$.

If one can return to the variable $y$ in obtained solutions, it is possible to obtain the solutions of the system $(7)$

$$
\begin{aligned}
& \omega(y, t)=e^{-\frac{l c_{3}}{4} \frac{2 y-l}{l}} \sum_{i=1}^{n+2} V_{i}^{n+\bar{i}}(y) G_{i}(t) \\
& p(y, t)=-\frac{2 c^{2}}{l} e^{-\frac{l c_{3}}{4} \frac{2 y-l}{l}} \sum_{i=1}^{n+2}\left(-\frac{l c_{3}}{4} V_{i}^{n+\bar{i}}(y)+U_{i-(-1)^{\bar{i}}}^{n-\bar{i}-(-1)^{\bar{i}}}(y)\right) \int_{0}^{t} G_{i}(t) d t+p(y, 0) .
\end{aligned}
$$

\section{Approximate solution}

We will find the product $V(x) G(t)$ in the form

$$
V(x) G(t)=\sum_{i=1}^{n+2} V_{i}^{n+\bar{i}}(x) \sum_{j=0}^{\infty} q_{i j} L_{j}(t),
$$

where $L_{j}(t)$ are Laggerr polynomials.

If $s \rightarrow \infty$, then

$$
\begin{gathered}
V_{x x}^{\prime \prime}(x) G(t)=\sum_{i=1}^{n} \frac{d^{2} V_{i}^{n+\bar{i}}(x)}{d x^{2}} \sum_{j=0}^{s} q_{i j} L_{j}(t)+\frac{d^{2} V_{n+1}^{n+1}(x)}{d x^{2}} \sum_{j=0}^{s} q_{n+1, j} L_{j}(t)+\frac{d^{2} V_{n+2}^{n}(x)}{d x^{2}} \sum_{j=0}^{s} q_{n+2, j} L_{j}(t) \\
V(x) G_{t t}^{\prime \prime}(t)=\sum_{i=1}^{n+2} V_{i}^{n+\bar{i}}(x) \sum_{j=0}^{s} q_{i, j+2} \sum_{k=0}^{j}(j+1-k) L_{k}(t) .
\end{gathered}
$$

From the boundary conditions $(11)$, considering that $V_{i}(-1)=V_{i}(1)=0[10,11]$, we have

$$
\frac{e^{\frac{l c_{3}}{4}} W(1, t)+e^{-\frac{l c_{3}}{4}} W(-1, t)}{2(n+1)^{2}}=\sum_{j=0}^{s} q_{n+2, j} L_{j}(t), \quad \frac{e^{\frac{l c_{3}}{4}} W(1, t)-e^{-\frac{l c_{3}}{4}} W(-1, t)}{2(n+2)^{2}}=\sum_{j=0}^{s} q_{n+1, j} L_{j}(t) .
$$

If we multiply the latter two equations by $e^{-t} L_{j}(t)$ and integrate with respect to $t$ from 0 to $\infty$ we can find the unknown coefficients $q_{n+1, j}, q_{n+2, j}, j=1, \ldots, s$ for (21). We substitute the expressions (22), (23) into the equation (14) and obtain

$$
\begin{array}{r}
\frac{4 c^{2}}{l^{2}}\left(\sum_{i=1}^{n} \frac{d^{2} V_{i}^{n+\bar{i}}(x)}{d x^{2}} \sum_{j=0}^{s} q_{i j} L_{j}(t)+\frac{d^{2} V_{n+1}^{n+1}(x)}{d x^{2}} \sum_{j=0}^{s} q_{n+1, j} L_{j}(t)+\frac{d^{2} V_{n+2}^{n}(x)}{d x^{2}} \sum_{j=0}^{s} q_{n+2, j} L_{j}(t)\right) \\
-\frac{c^{2} A_{3}^{2}}{4} \sum_{i=1}^{n+2} V_{i}(x) \sum_{j=0}^{s} q_{i j} L_{j}(t)-\sum_{i=1}^{n+2} V_{i}(x) \sum_{j=0}^{s} q_{i, j+2} \sum_{k=0}^{j}(j+1-k) L_{k}(t)=0
\end{array}
$$


The equation (24) is multiplied by $\frac{\bar{V}_{i}(x)}{\sqrt{1-x^{2}}} e^{-t} L_{j}(t), i=1, \ldots, n, j=1, \ldots, s$ and integrated with respect to $t$ from 0 to $\infty$ and with respect to $x$ from -1 to 1 . We obtain $i=n$ of the systems, each of them contains $j=s$ of equations and $j=s$ of the unknowns, from which we can find the rest of unknown coefficients $q_{i, j}, i=1, \ldots, n, j=1, \ldots, s$ for $(21)$. Thus, we have found the solution of the system (9) on the interval $x \in[-1,1]$, namely

$$
\begin{aligned}
W(x, t) & =e^{-\frac{l c_{3}}{4} x} \sum_{i=1}^{n+2} V_{i}^{n+\bar{i}}(x) \sum_{j=0}^{s} q_{i j} L_{j}(t), \\
P(x, t) & =-\frac{2 c^{2}}{l} e^{-\frac{l c_{3}}{4} x} \sum_{i=1}^{n+2}\left(-\frac{l c_{3}}{4} V_{i}^{n+\bar{i}}(x)+U_{i-(-1)^{\bar{i}}}^{n-\bar{i}-(-1)^{\bar{i}}}(x)\right) \sum_{j=0}^{s} q_{i j}\left[L_{j}(t)-L_{j+1}(t)\right]+P(x, 0),
\end{aligned}
$$

where $U_{n+2}^{n}(x)=T_{n+2}^{\prime \prime}(x), U_{n+1}^{n-1}(x)=T_{n+1}^{\prime \prime}(x)$. We return to the variable $y$ in the obtained solutions and get the solutions of the system (7)

$$
\begin{aligned}
& \omega(y, t)=e^{-\frac{l c_{3}}{4} \frac{2 y-l}{l}} \sum_{i=1}^{n+2} V_{i}^{n+\bar{i}}(y) \sum_{j=0}^{s} q_{i j} L_{j}(t), \\
& p(y, t)=-\frac{2 c^{2}}{l} e^{-\frac{l c_{3}}{4} \frac{2 y-l}{l}} \sum_{i=1}^{n+2}\left(-\frac{l c_{3}}{4} V_{i}^{n+\bar{i}}(y)+U_{i-(-1)^{\bar{i}}}^{n-\bar{i}-(-1)^{\bar{i}}}(y)\right) \sum_{j=0}^{s} q_{i j}\left[L_{j}(t)-L_{j+1}(t)\right]+p(y, 0) .
\end{aligned}
$$

The results of the solution of the system of equations (7) for $l=100000 \mathrm{~m}, \lambda=0.01, z=0.908$, $R=500 \mathrm{Joul} /(\mathrm{kg} \cdot \mathrm{K}), D=1.4 \mathrm{~m}, T=300 \mathrm{~K}, q_{0}=894 \mathrm{~m}^{3} / \mathrm{s}, q_{0 n}=993 \mathrm{~m}^{3} / \mathrm{s}, q_{l}=894 \mathrm{~m}^{3} / \mathrm{s}, q_{l n}=$ $993 \mathrm{~m}^{3} / \mathrm{s}, p_{0}=70 \mathrm{~atm}, p_{1}=58.4 \mathrm{~atm}, \rho_{s}=\rho_{0}=0.682 \mathrm{~kg} / \mathrm{m}^{3}, v_{1}=6 \mathrm{~m} / \mathrm{s}, v_{2}=12 \mathrm{~m} / \mathrm{s}, \gamma_{0}=0.00069$, $\gamma_{l}=0.00075, c=500 \mathrm{~m} / \mathrm{s}, \Delta x=5000 \mathrm{~m}, \Delta t=420 \mathrm{~s}$.

Table 1. Value of mass consumption for the pipeline with length $l=100000 \mathrm{~m}$ at different values of time $t$ and the coordinate $y_{1}$ for analytical-approximate solution of the problem from the Item 4 and the coordinate $y_{2}$ for approximate solution of the task from the Item 5 at $n=10$, the value $t$ is indicated in seconds in Table.

\begin{tabular}{|c|c|c|c|c|c|c|c|c|}
\hline \multirow{2}{*}{$t$} & \multicolumn{9}{|c|}{$y$} \\
\cline { 2 - 9 } & \multicolumn{2}{|c|}{10000} & \multicolumn{2}{c|}{30000} & \multicolumn{2}{c|}{60000} & \multicolumn{2}{|c|}{90000} \\
\cline { 2 - 9 } & $y_{1}$ & $y_{2}$ & $y_{1}$ & $y_{2}$ & $y_{1}$ & $y_{2}$ & $y_{1}$ & $y_{2}$ \\
\hline 420 & 407.22 & 407.22 & 407.42 & 407.42 & 407.68 & 407.68 & 407.87 & 407.87 \\
\hline 1260 & 421.70 & 421.70 & 421.99 & 421.99 & 422.40 & 422.40 & 422.77 & 422.77 \\
\hline 2100 & 429.77 & 429.77 & 430.02 & 430.02 & 430.39 & 430.39 & 430.74 & 430.74 \\
\hline 2940 & 434.26 & 434.26 & 434.46 & 434.46 & 434.74 & 434.74 & 435.01 & 435.01 \\
\hline 3780 & 436.77 & 436.77 & 436.91 & 436.91 & 437.10 & 437.10 & 437.30 & 437.30 \\
\hline 4620 & 438.17 & 438.17 & 438.26 & 438.26 & 438.39 & 438.39 & 438.52 & 438.52 \\
\hline 5460 & 438.95 & 438.95 & 439.01 & 439.01 & 439.09 & 439.09 & 439.18 & 439.18 \\
\hline 6300 & 439.38 & 439.38 & 439.42 & 439.42 & 439.47 & 439.47 & 439.53 & 439.53 \\
\hline 7140 & 439.63 & 439.63 & 439.65 & 439.65 & 439.68 & 439.68 & 439.72 & 439.71 \\
\hline 7980 & 439.76 & 439.76 & 439.78 & 439.77 & 439.80 & 439.79 & 439.82 & 439.81 \\
\hline 8820 & 439.84 & 439.81 & 439.85 & 439.80 & 439.86 & 439.79 & 439.87 & 439.78 \\
\hline 9660 & 439.88 & 439.70 & 439.89 & 439.60 & 439.89 & 439.43 & 439.90 & 439.26 \\
\hline
\end{tabular}


Table 2. Value of pressure for the pipeline with length $l=100000 \mathrm{~m}$ at different values of time $t$ and the coordinate $y_{1}$ for analytical-approximate solution of the problem from the Item 4 and the coordinate $y_{2}$ for approximate solution of the task from the Item 5 at $n=10$, the value $t$ is indicated in seconds in Table.

\begin{tabular}{|c|c|c|c|c|c|c|c|c|}
\hline \multirow{3}{*}{$t$} & \multicolumn{7}{|c|}{$y$} \\
\cline { 2 - 9 } & \multicolumn{2}{|c|}{10000} & \multicolumn{2}{|c|}{30000} & \multicolumn{2}{|c|}{60000} & \multicolumn{2}{|c|}{90000} \\
\cline { 2 - 9 } & $y_{1}$ & $y_{2}$ & $y_{1}$ & $y_{2}$ & $y_{1}$ & $y_{2}$ & $y_{1}$ & $y_{2}$ \\
\hline 420 & 68.92 & 68.92 & 66.73 & 66.73 & 63.31 & 63.31 & 59.68 & 59.68 \\
\hline 1260 & 68.89 & 68.89 & 66.71 & 66.71 & 63.28 & 63.28 & 59.66 & 59.66 \\
\hline 2100 & 68.86 & 68.86 & 66.68 & 66.68 & 63.26 & 63.26 & 59.64 & 59.64 \\
\hline 2940 & 68.84 & 68.84 & 66.65 & 66.65 & 63.23 & 63.23 & 59.62 & 59.62 \\
\hline 3780 & 68.82 & 68.82 & 66.64 & 66.64 & 63.22 & 63.22 & 59.60 & 59.60 \\
\hline 4620 & 68.81 & 68.81 & 66.63 & 66.63 & 63.21 & 63.21 & 59.59 & 59.59 \\
\hline 5460 & 68.80 & 68.80 & 66.62 & 66.62 & 63.20 & 63.20 & 59.58 & 59.58 \\
\hline 6300 & 68.80 & 68.80 & 66.61 & 66.61 & 63.19 & 63.19 & 59.58 & 59.58 \\
\hline 7140 & 68.80 & 68.79 & 66.61 & 66.61 & 63.19 & 63.19 & 59.58 & 59.58 \\
\hline 7980 & 68.79 & 68.78 & 66.61 & 66.61 & 63.19 & 63.19 & 59.57 & 59.58 \\
\hline 8820 & 68.79 & 68.77 & 66.61 & 66.61 & 63.19 & 63.19 & 59.57 & 59.59 \\
\hline 9660 & 68.79 & 68.75 & 66.61 & 66.61 & 63.19 & 63.19 & 59.57 & 59.60 \\
\hline
\end{tabular}

\section{Conclusions}

The obtained results confirm effective usage of the built biorthogonal polynomials for solving problems of the mathematical physics. Not only the number of products $n$ of the corresponding series sum, accuracy of calculation have an essential influence on finding of approximate solution of our problem, but the method of the function by time. If one can find the function by time by means of the analytical method, then we obtain analytical-approximate solution from the Item 4, which helps us to find the solution of system (7) on the random period of time, contrary to the approximate solution from the Item 5, which will be correct only comparable on small intervals of time $t \leqslant 2.5$ hour, where the function concerning time is found through the approximate method.

[1] Abramovich M., Stygan I. Reference book of Special Functions with Formulae, Diagrams and Mathematical Tables. Moscow, Science (1979).

[2] Gletcher K. Numerical Methods of the Base of the Galierkin's Method. Moscow, World (1988).

[3] Dziadyk V.K. Approximate Methods of Solution of Differential and Integral Equations. Kyiv, Scientific Thought (1998).

[4] Dziadyk V.K. Introduction in to the Theory of Uniform Approximation of Functions by Polynomials. Moscow, Science (1977).

[5] Kantorovich L. V., Akilov G. P. Functional Analysis. Moscow, Science (1984).

[6] Korneychuk N. P. Accurate Constants in the Theory of Approximation. Moscow, Science (1987).

[7] Lantsosh K. Practical Methods of Applied Analysis. Moscow, State Publishing House of PhysicalMathematical Literaure (1961).

[8] Ditkin V. A., Prudnikov A. P. Operational Calculation. Moscow, Higher School (1975).

[9] Lopukh N., Pritula M., Pjanylo Ya., Savula Ya. Algorithms of Calculation of Hydrodynamical Parameters of Gas Flow in Pipelines. Visnyk of Lviv University. Serie of Appl. Math. and Comp. Sci, 12, 108-117 (2007) (in Ukrainian).

[10] Pyanylo Ya. D., Sobko V. G. Building and Research of Biorthogonal Polynomials on the Base of the Chebyshev Polynomials. Appl. Problems of Mech. and Math. 11, 135-141 (2013) (in Ukrainian). 
[11] Pyanylo Ya., Sobko V. Research of Peculiarities of Spectral Schedules in the Bases of Orthogonal, Quaziorthogonal, Biorthogonal Polynomials. Phisical-Mathematical Modelling and Information Techologies. 19, 146-156 (2014) (in Ukrainian).

[12] Ghoreishi F., Mohammad Hosseini S. The Tau method and a new preconditioner. J. Comp. Appl. Math. 163(2), 351-379 (2004).

[13] Coutsias E. A., Hagstorm T., Hesthaven J. S., Torres D. Integration preconditioners for differential operators in spectral $\tau$-methods. Proc. 3rd International Conference on Spectral and High Order Methods, Houston, TX, 21-38 (1995).

[14] Bernardi C., Maday Y. Properties of some weighted sobolev spaces, and applications to spectral approximations. SIAM J. Numer. Anal. 26, 769-829 (1989).

[15] Jie Shen. Efficient spectral-Galerkin method II. Direct solvers for second- and fourthorder equations by using Chebyshev polynomials. SIAM J. Sci. Comput. 16, 74-87 (1995).

[16] Jie Shen. Efficient Chebyshev-Legendre Galerkin methods for elliptic problems. In A. V. Ilin and R. Scott, editors, Proceedings of ICOSAHOM'95. Houston. J. Math. 233-240 (1996).

[17] Badkov V. M. Convergence in the mean and almost everywhere of Fourier series in polynomials orthogonal on an interval. Math. USSR Sbornik. 2, MR0355464 (50:7938) 223-256 (1974).

[18] John P. Boyd. Chebyshev and Fourier Spectral methods, 2nd edition. Dover Publication, Inc., Mineola, New York (2001).

[19] Walte A. Strauss. Partial Differential Equations: An Introduction, 2nd edition, John Wiley and Sons (2008).

[20] Cabos Ch. A preconditioning of the tau operator for ordinary differential equations. Z. Angew. Math. Mech. 74 (11), 521-532 (1994).

[21] Coutsias E. A., Hagstorm T., Hesthaven J., Torres D. Integration preconditioners for differential operators in spectral $\tau$-methods. Proc. 3rd International Conference on Spectral and High Order Methods, Houston, TX, 21-38 (1995).

\title{
Методи знаходження розподілу тиску в трубопроводі
}

\author{
П'янило Я., Собко В. \\ Центр математичного моделювання \\ Інституту прикладних проблем механіки і математики \\ ім. Я. С. Підстригача НАН України \\ вул. Дудаєва, 15, 79000, Львів,Україна
}

\begin{abstract}
У праці на базі побудованих авторами біортогональних поліномів запропоновано метод розв'язування задач математичної фізики, зокрема для розрахунку нестаціонарного руху газу в трубопроводах. Досліджено спосіб розв'язування задачі методом розділення змінних у базисі біортогональних поліномів. Знайдено аналітичнонаближений та наближений розв'язки задачі у вигляді суми ряду біортогональних та квазіспектральних поліномів. Проведено порівняльний аналіз між отриманими наближеним та аналітично-наближеним розв'язками. Вивчено вплив параметрів методів, зокрема порядку часткової суми, розрядної сітки та похибки обчислення на точність отриманого розв'язку. Результати обчислень подано у вигляді таблиць.
\end{abstract}

Ключові слова: спектральні методи, математична модель, нестачіонарний рух газу, лінеаризачіл, біортогональні та квазіортогональні поліноми.

2000 MSC: $12 \mathrm{E} 10$

удк: 519.6:539.3 